\title{
LIPID AND PHOSPHOLIPID COMPOSITION OF NON-PIGMENTED AND PIGMENTED HYPHAL CELL WALLS OF (-) STRAIN OF BLAKESLEA TRISPORA
}

\author{
MICHEL S. CHENOUDA \\ Biochemistry Department, National Research Center, \\ Dokki, Cairo, Egypt
}

(Received February 3, 1972)

\begin{abstract}
Exposure of the hyphae of (-) strain of Blakeslea trispora to air greatly stimulated the accumulation of carotenes and induced changes in the composition of the cell wall lipid. Fractionation of wall lipid revealed the presence of different lipid classes (phospholipids, monoglycerides, diglycerides, ergosterol, free fatty acids, triglycerides, and waxes). Phospholipids, free fatty acids, and waxes constitute the major part of wall lipid. The difference in wall lipid between non-pigmented and pigmented hyphae was quantitative rather than qualitative.

The phospholipid fraction mainly contained phosphatidic acid, diphosphatidylglycerol, glucosylphosphatidylglycerol, and a trace of phosphatidylglycerol. No qualitative or quantitative difference was observed in the phospholipid components of the phospholipid fractions.
\end{abstract}

NickERSON ( 1 ) called attention to the possible role of lipids in the architecture of the yeast cell walls. Although many of the recent investigations have involved the characterization of lipids from whole microbial cells, it is now generally recognized that the bulk of the cellular lipid is localized in the membrane systems $(2-4)$. However, studies on the lipid content of biological membranes are still in their infancy, and the informations available are fragmentary and restricted to a few bacterial membranes.

The changes in the lipid composition of Staphylococcus aureus membranes accompanying transfer of cells from anaerobic to aerobic conditions were reported by FRERMAN and WHITE (4). LASCELLES and SzILÁGYI ( 2 ) suggested that the formation of photosynthetic pigments in Rhodopseudomonas spheroides was accompanied by modifications in the membrane structure. In addition, the biological membranes of Mycoplasma laidlawii can withstand most pronounced qualitative and quantitative changes in its lipid component without losing its vital physiological functions (5).

The changes in the phospholipid composition of mycelia of Blakeslea trispora were studied with reference to the influence of sex, mycelial age, 
and addition of $\beta$-factor $(6,7)$. The earlier reports $(8,9)$ showed that exposure of the (-) strain of Bl. trispora to air was accompanied by a remarkable accumulation of carotenes and a striking change in the thickness and chemical composition of the cell walls. The difference in the amounts of carbohydrates and lipids was clear. This paper reports the results of detailed studies on the composition and structure of lipid and phospholipid fractions of the cell walls of the non-pigmented and the pigmented hyphae of $(-)$ strain of $B l$. trispora.

\section{MATERIALS AND METHODS}

General Cultural Conditions. Blakeslea trispora Thaxter (-) strain (CBS 131.59) was obtained from the Centraalbureau voor Schimmelcultures, Baarn, Holland. Culture in a synthetic liquid medium was carried out in two different ways as previously described by CHENOUdA (9). The organism gave white fluffy hyphae when cultured submerged and yellow pigmented rigid hyphae when cultured exposed to air.

Isolation and Purification of Cell Walls. Cell wall fragments of both the non-pigmented and the pigmented hyphae were prepared and purified according to the methods adopted by CHENOUDA ( 9 ). The percentage breakage of the cell walls was more than $95 \%$ by examining the cell wall preparations under a phase-contrast microscope. The purified cell wall preparations were freeze-dried and stored at $-15^{\circ}$.

Extraction of Lipids. The lipids were extracted from the cell walls with chloroform-methanol $(2: 1)$ according to the method adopted by CHENOUDA (10). Chloroform extracts were washed (11) and evaporated under a reduced pressure. Flasks containing the purified lipid fractions were desiccated over $\mathrm{P}_{2} \mathrm{O}_{5}$ to a constant weight. The percentage of lipids was then calculated and raised to a known volume with chloroform. Samples of these solutions were used for various analysis.

Fractionation, Identification, and Quantitative Determination of Lipids. The separation of lipids to classes was conducted on glass plates coated with 250-270 $\mu$ m layers of Stahl silica gel G. A known amount of lipid in chloroform (with reference materials) was applied as a streak to the activated silica gel plates and developed with a solvent system of hexane-ether-acetic acid $(8: 2: 1)$. The development of the plates in this way resulted in very distinct bands of different lipid classes. The phospholipids, whose band did not migrate from the origin, were collected from the plates by scraping the silica gel. After removal of the phospholipid bands, the plates were exposed to iodine vapour for $1 \mathrm{~min}$. The individual bands were separated by cutting parallel channels with spatula end and the lipid in various bands collected and weighed according to the method of KOMAREK et al. (12). Chloroformmethanol-water mixture $(65: 40: 5)$ was used for the collection of phospho- 
lipids ( 13 ) and ether was used for the elution of different lipid fractions from the silica gel.

In some cases, plates were sprayed with Rhodamine $6 \mathrm{G}$ and viewed under ultraviolet ray for the detection and comparison of different lipid materials.

Fractionation, Identification, and Quantitative Determination of Phospholipids. Fractionation of phospholipids was affected by thin layers of silica gel $\mathrm{G}$ and silica gel $\mathrm{H}$. Solvent systems for the former were (i) chloroformmethanol-water $(65: 25: 4)$, (ii) chloroform-methanol-acetic acid-water (100: $50: 14: 6$ ), (iii) chloroform-methanol-acetic acid-water $(125: 37: 10: 2)$, and (iv) chloroform-methanol-7 $\mathrm{N}$ ammonia $(65: 35: 5)$. For silica gel $\mathrm{H}$, the solvent systems were (v) chloroform-methanol-water-acetic acid $(65: 25: 4: 1)$, and (vi) chloroform-methanol-4.5 $\mathrm{N}$ ammonia $(65: 30: 5.7)+$ hydrogencarbonate to $\mathrm{pH} 7$.

After the plates were developed, they were air dried and phospholipids were stained with (a) iodine vapour, (b) ammonium molybdate (10), (c) Dragendorff's reagent for choline-containing phospholipids (13), (d) phosphomolybdic blue stain for choline-containing phospholipids (10), (e) $0.2 \%$ Ninhydrin in ethanol for amino group-containing phospholipids, and (f) periodicSchiff reagent for compounds having vicinal hydroxyl groups (14).

Quantitative determination of different phospholipids was carried out by applying the phospholipid fraction as a streak on layers of silica gel $G$ and developed with chloroform-methanol-water $(65: 25: 4)$. The plates were air dried and exposed to iodine vapour for a few seconds. The bands were eluted and phospholipids were estimated as described for the determination of different lipid classes.

Hydrolysis and Analysis of Phospholipids. Deacylation of different phospholipids and chromatography of the hydrolysis products were affected by the methods adopted by Dos SanTos MOTA et al. (15). Spot tests included the molybdate reagent for phosphate and Ninhydrin for amino-containing compounds.

Acid hydrolysis for the study of carbohydrate-containing phospholipid was effected with $3 \mathrm{~N} \mathrm{HCl}$. The hydrolyzate was dried and passed through a column filled with $5 \mathrm{ml}$ of Dowex-I $\left(\mathrm{OH}^{-}\right)$underneath $5 \mathrm{ml}$ of Dowex-50W $\left(\mathrm{H}^{+}\right)$. The eluate was evaporated to dryness and the residue was dissolved in water. Carbohydrates were identified by using reference compounds, by paper chromatography with solvent systems of butanol-pyridine-water $(5: 3: 2)$ and ethyl acetate-acetic acid-water $(9: 2: 2)$. The chromatograms were air dried and developed with ammoniacal silver nitrate.

Assay Procedures. Established methods were used for the microassay of phosphorus (16), glycerol (17), and glucose (18).

\section{RESULTS}

Cell wall fractions of Blakeslea trispora, cultured submerged (non-pigmented) 
Table 1. Quantity and composition of wall lipids of non-pigmented and pigmented hyphae of (-) strain of Blakeslea trispora.

\begin{tabular}{l|c|c}
\hline \multirow{2}{*}{ Lipid material } & \multicolumn{2}{|c}{ Free lipid (\%) } \\
\cline { 2 - 3 } & Non-pigmented & Pigmented \\
\hline Phospholipids & 28.1 & 20.2 \\
Monoglycerides & 7.3 & 5.7 \\
Diglycerides & 2.0 & 6.1 \\
Ergosterol & 4.2 & 7.9 \\
Free fatty acids & 23.4 & 30.1 \\
Triglycerides & 9.1 & 8.9 \\
Waxes & 26.0 & 21.0 \\
\hline
\end{tabular}

or exposed to air (pigmented), were prepared and freeze-dried as described. These preparations were white-grey and microscopically consisted of cell wall fragments of different sizes. On careful examination with a phase-contrast microscope an occasional intact cell or cytoplasmic particle was detected.

\section{Lipids of Cell Walls}

A known weight $(0.8-1.0 \mathrm{~g})$ of freeze-dried cell wall preparation was exhaustively extracted with chloroform-methanol $(2: 1)$. Under these conditions, the total lipids accounted for $28-30 \%$ of the cell wall in dry weight of the non-pigmented hyphae and $13-15 \%$ of the cell wall in dry weight of the yellow hyphae of (-) strain of $B l$. trispora.

Fractionation of cell wall lipids of non-pigmented and pigmented hyphae revealed the presence of 7 different classes of lipids (Fig. 1) with a minute amount of carotenes. A mixture of hexane-diethylether-acetic acid $(8: 2: 1)$ was the best of all the solvents tried. After the developing of the plates, they were air dried and examined under UV ray and then sprayed with Rhodamin $6 \mathrm{G}$ or exposed to iodine vapour. Waxes with carotenes migrated beyond the solvent front followed by triglycerides, free fatty acids, sterols, diglycerides, monoglycerides, and phospholipids. The latter did not migrate from the origin. No qualitative difference was observed in the cell wall lipid pattern between the non-pigmented and the pigmented hyphae (Fig. 1).

The band corresponding to the sterol was eluted and purified on silica gel plates. From the UV spectrum and behaviour in different solvent systems, it was concluded that ergosterol is the main component of the sterol fraction of the cell wall lipid.

The percentage distributions of different lipid classes in the cell wall lipids of non-pigmented and pigmented hyphae are given in Table 1 . These data show that the phospholipids, free fatty acids, and waxes constitute the major amount of wall lipid of Bl. trispora, comprising $78 \%$ of the total lipid in the 


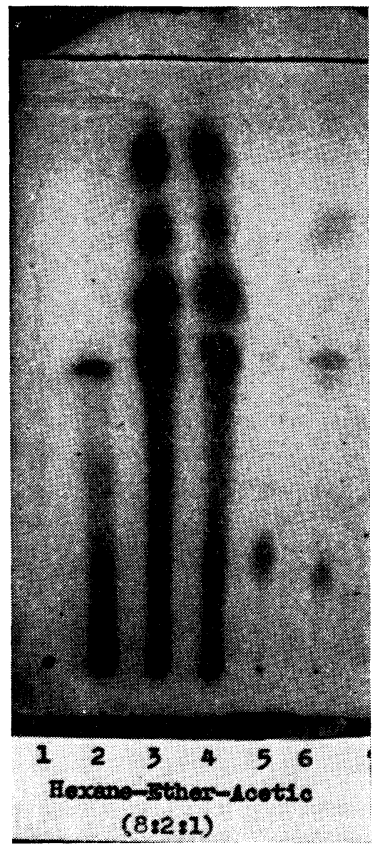

Fig. 1. Chromatoplate of wall lipids of non-pigmented and pigmented hyphae of (-) strain of Blakeslea trispora.

The plate was developed with hexane-ether-acetic acid $(8: 2: 1)$, and exposed to iodine vapour.

1: Standard mixture of lecithin and cephalin. 2: Standard ergosterol. 3: Wall lipids of non-pigmented cells. 4: Wall lipids of pigmented cells. 5: Standard monostearate. 6: Standard mixture of monostearate, dipalmitin, ergosterol and tripalmitin.

non-pigmented hyphae and $71 \%$ in the pigmented hyphae. Other components are present in a relatively smaller amount. However, it is clear that the percentages of phospholipids and waxes in the wall lipid of non-pigmented hyphae are higher than those of the wall lipid of pigmented hyphae. On the other hand, the amounts of diglycerides and ergosterol are relatively higher in the wall lipid of pigmented hyphae. No striking difference was observed in the amounts of monoglycerides and triglycerides.

\section{Phospholipids of Cell Walls}

Phospholipid bands which did not migrate from the origin were eluted and resolved on silica gel layers using different solvent systems. The compounds were identified from their $R f$ values in different solvent systems, specific colour reaction, $R f$ values of water-soluble deacylation products, prod- 


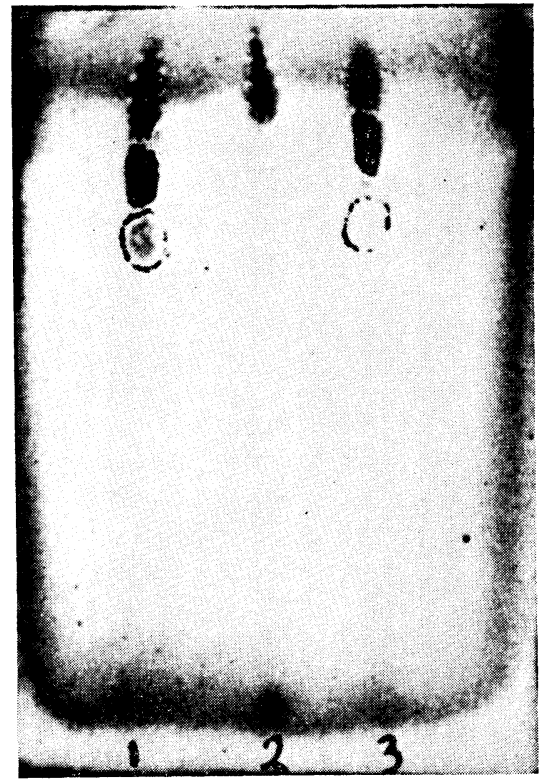

Fig. 2. Chromatoplate of non-pigmented and pigmented wall phospholipids of (-) strain of Blakeslea trispora.

The plate was developed with chloroform-methanol-water (65: $25: 4)$, and stained with ammonium molybdate.

1: Wall phospholipids of non-pigmented cells. 2: Standard phosphatidic acid. 3: Wall phospholipids of pigmented cells.

ucts of acid hydrolysis, and the phosphorus/glycerol ratio. Three principal phospholipids were detected (Fig. 2) with a trace spot of phospholipid which was speculated as phosphatidylglycerol.

Compound $(A)$ : Phosphatidic acid. It reacted with ammonium molybdate and gave the same $R f$ value as the standard phosphatidic acid in different solvents. It released a compound comparable to $\alpha$-glycerophosphate upon mild alkaline hydrolysis. The estimated phosphorus/glycerol ratio had a mean value around $1: 1$.

Compound $(B)$ : Diphosphatidylglycerol. It reacted with ammonium molybdate. It gave positive reaction with periodate-Schiff reagent, indicating the presence of vicinal hydroxyl groups. After deacylation it gave a watersoluble phosphate ester with an $R f$ value comparable to bis-(glycerylphosphoryl) glycerol. The estimated phosphorus/glycerol ratio had a mean value of $1: 1.6$.

Compound $(C)$ : Glucosylphosphatidylglycerol. It reacted with ammonium molybdate. It gave a weak positive reaction with periodate-Schiff reagent. Deacylation of this compound gave a water-soluble phosphate ester with an 
Table 2. Characterization and estimates of phospholipid components of non-pigmented and pigmented hyphae wall phospholipid fractions of (-) strain of Blakeslea trispora.

\begin{tabular}{c|cc|c|c|c|c|c|c|c} 
& Spot & $\begin{array}{c}R f X^{*} \text { Amm. } \\
100\end{array}$ & moly. & Schiff & Dragen. & Phosph. & Ninhyd. & Deacylation** & \multicolumn{2}{|c|}{$\begin{array}{c}\text { Phospholipid (\%) } \\
\text { Non- }\end{array}$} & Pigmented & Pigmed \\
\hline A & 95 & + & - & - & - & - & GP & 43.1 & 42.8 \\
B & 84 & + & + & - & - & - & GPGPG & 34.6 & 33.3 \\
C & 72 & + & + & - & - & - & GPG & 22.3 & 23.8 \\
\hline
\end{tabular}

* Solvent system: Chloroform-methanol-water (65:25:4).

** GP, $\alpha$-glycerophosphate; GPGPG, bis-(glycerolphosphoryl) glycerol ; GPG, glycerylphosphorylglycerol.

$R f$ value comparable to glycerylphosphorylglycerol. Glucose was the only sugar detected after acid hydrolysis. The estimated phosphorus/glycerol/glucose ratio had a mean value $1: 2.1: 1.1$. Concerning the glucose-glycerol linkage, the $\mathrm{C}-1$ of glucose seems to be blocked since the intact compound. did not give positive reaction with alkaline silver nitrate.

The characterization and the quantitative estimates of the main phospholipids in the wall lipids of non-pigmented and pigmented hyphae of $\mathrm{Bl}$. trispora are presented in Table 2 . The results show that phosphatidic acid and cardiolipin constitute more than $75 \%$ of the phospholipid fraction. No qualitative or quantitative difference is shown in the phospholipid pattern of the phospholipid fractions of non-pigmented and pigmented hyphae.

\section{DISCUSSION}

Mated cultures of Blakeslea trispora, but not (+) or (-) strain alone, produce a considerable amount of carotenes in addition to $\beta$-factor which when added to the culture medium of $(-)$ strain greatly stimulate carotenogenesis (19). However, Chenouda et al. (8) and CHenouda (9) found that the striking accumulation of carotenes on exposure of the hyphae of $(-)$ strain to air was accompanied by noticeable change in wall lipids. The pigmented cell wall contains a significantly less free lipid than the cell wall of the non-pigmented hyphae. The carotene synthesis of the fungus was raised from $150 \mu \mathrm{g} /$ liter in submerged culture to $1040 \mu \mathrm{g} /$ liter in the cultures. exposed to air. FRERMAN and WHITE (4) reported a marked change in the lipid composition of Staphylococcus aureus accompanying the transfer of cells from anaerobic to aerobic condition. AMES (20) mentioned that the lipids are the object of attention because the membrane delimiting the cell is supposed to be constituted largely of lipid. Furthermore, since the bulk of the cellular lipid is located in the membrane systems of bacteria (2-4), it was 
thought of interest to study in detail the composition and structure of the lipid and phospholipid fractions of the cell walls of non-pigmented (submergically cultured) and pigmented (exposed to air) hyphae of (-) strain of $B l$. trispora. In spite of the reasonable informations on the free lipid composition of cell membranes of bacteria, very little informations are available on the free lipid composition of cell walls isolated from fungi.

The present studies indicate that phospholipids, monoglycerides, diglycerides, ergosterol, free fatty acids, triglycerides, and waxes are the main lipid classes in the cell wall of $(-)$ strain of $B l$. trispora. The quantitative determination of each class of lipids shows that phospholipids, waxes, and free fatty acids are the major components of the lipid fractions. However, phospholipids and waxes are the most abundant components in the wall lipid of non-pigmented hyphae but free fatty acids and waxes are the most abundant in the wall lipid of pigmented hyphae. In addition, the wall lipid of nonpigmented hyphae has a significantly less carotenes, diglycerides, and ergosterol. Therefore, it seems that the wall lipid difference between non-pigmented and pigmented hyphae is quantitative rather than qualitative.

These studies failed to indicate the presence of choline- or ethanolaminecontaining phospholipids in the wall lipid of (-) strain of Bl. trispora. Chromatographic and staining characteristic and examination of hydrolytic products revealed the presence of phosphatidic acid, diphosphatidylglycerol, and glucosylphosphatidylglycerol as the main phospholipid components of the wall lipids. A trace spot obtained with a more sensitive Schiff reagent corresponding to phosphatidylglycerol was detected. The quantitative data revealed that phosphatidic acid is the most abundant and cardiolipin is the next most abundant phospholipids. Glucosylphosphatidylglycerol represents approximately $23 \%$ of the phospholipid fraction. However, no quantitative difference was observed in the components of the phospholipid fractions isolated from wall lipids of non-pigmented and pigmented cells.

In regard to the identity of phospholipid (C), it was concluded that it is a derivative of phosphatidylglycerol, since a mild alkaline hydrolysis furnished glycerophosphorylglycerol. The compound was hydrolyzed with $\mathrm{HCl}$ and the water-soluble hydrolyzate was chromatographically investigated. Only one spot corresponding to standard glucose was detected. A further detailed study was made on its chemical structure. The estimated analysis of phosphorus/ glycerol/glucose ratio showed a mean value around $1: 2: 1$. In addition, C-1 of glucose seems to be blocked because the intact compound did not give a positive reaction with alkaline silver nitrate. From all these analyses and from its chromatographic behaviour in different solvent systems, it is postulated that it is glucosylphosphatidylglycerol. The presence of phosphatidylglucose in the cell membrane of Mycoplasma laidlawai ( 5) and a glucosamine derivative of phosphatidylglycerol in the cell membrane of Bacillus megaterium (21) has been reported.

Preliminary evidence concerning the biosynthetic pathway of cardiolipin 
showed that the intact molecule of phosphatidylglycerol is transformed in vitro to cardiolipin by the addition of a new phosphatidic acid moiety (22). Phosphatidylglycerol is the most metabolically active phospholipid in many microorganisms $(2,22,23)$ which may account for the trace amount detected in the cell walls.

Possibly, it can be deduced from this and from the previous studies ( 8 , 9 ) that the submerged hyphae have a wall structure differing from that exposed to air and therefore induced biochemically different forms of the organism.

The author is greatly indebted to Dr. E. W. Jwanny for her helpful discussions.

\section{REFERENCES}

1) W.J. NicKerson, Bacteriol. Rev., 27, 305 (1963).

2) J. Lascelles and J.F. Szilágyi, J. Gen. Microbiol,, 38, 55 (1965).

3) K.Y. Cho and M.R.J. Salton, Biochim. Biophys. Acta, 116, 73 (1966).

4) F.E. Frerman and D.C. White, J. Bacteriol., 94, 1868 (1967).

5) I. KahANe and R. Razin, Biochim. Biophys. Acta, 183, 79 (1969).

6) G.A. Bayadzhan, L.A. Vakulova, É.G. Dedyukhina, M.N. Bekhtereva and G.I. SAMOKHVALOV, Mikrobiologiya, 37, 670 (1968).

7) L.A. Vakulova, G.A. Bayodzhan, M.I. Bekhtereva and G.I. Samokhvalov, Dokl. Akad. Nauk SSSR, 191, 69 (1970).

8) M.S. Chenouda, G. BRitton and T.W. Goodwin, in preparation for publication.

9) M.S. Chenouda, J. Gen. Appl. Microbiol., 18, 143 (1972).

10) M.S. Chenouda, J. Gen. Appl. Microbiol., 16, 501 (1970).

11) J. Folch, M. Lees and G.H. Sloan-Stanley, J. Biol. Chem., 226, 497 (1957).

12) R.J. Komarek, R.G. Jensen and B.W. Pickett, J. Lipid Res., 5, 268 (1964).

13) A. Sheltawy and R.M.C. Dawson, Chromatographic and Electrophoretic Techniques, ed. Ivor Smith, 2nd. ed., William Heinemann Medical Books Ltd., England (1969), vol. 1, p. 450.

14) J. Baddiley, J.G. Buchanan, R.E. Handschumacher and J.F. Prescott, J. Chem. Soc., 2818 (1956).

15) J.M. Dos Santos Mota, J.A.F. op Den Kamp, H.M. VerheiJ and L.L.M. vaN DEENEN, J. Bacteriol., 104, 611 (1970).

16) W.R. Morrison, Anal. Biochem., 7, 218 (1964).

17) A. Jover, J. Lipid Res., 4, 228 (1963).

18) F.W. Yemm and A.J. Willis, Biochem. J., 57, 508 (1954).

19) R.P. SutTer and M.E. Rafelson, J. Bacteriol., 95, 426 (1968).

20) G.F. AMES, J. Bacteriol., 95, 833 (1968).

21) J.A.F. op Den Kamp, U.M.T. Houtsmuller and L.L.M. van Deenen, Biochim. Biophys. Acta, 106, 438 (1965).

22 ) N.Z. Stanacev, Y.Y. Change and E.P. Kennedy, J. Biol. Chem., 242, 3018 (1967).

23) L.L.M. van Deenen and G.H. De HaAs, Ann. Rev. Biochem., 35, 157 (1966). 